\title{
Perceptual Development and Category Generalization
}

\author{
Linda B. Smith \\ Indiana University
}

\begin{abstract}
Smith, Linda B. Perceptual Development and Category Generalization. Child Development, $1979,50,705-715$. This work is concerned with developmental changes in the structure of classifications. The central claim is that young children's undifferentiated perceptions of complex stimuli are highly structured by wholistic similarity whereas older children's perceptions are structured by component dimensions. It is shown in 2 experiments that young children systematically and spontaneously generalize a category if it is well organized by overall similarity but not if it is organized by a criterial dimension. Older children, on the other hand, spontaneously apprehend and extend a category by its dimensional structure. The third experiment was designed to test the hypothesis that criterial property categories are preferred in classification tasks requiring the explicit discovery of a general rule. It was found that younger children's attention to the dimensional relations within a category increases under rule-discovery instructions, although they still have difficulty ignoring wholistic similarity relations. The trend from similarity to dimensional classification is discussed in the context of Piagetian classification tasks and familyresemblance accounts of natural categories.
\end{abstract}

A major principle of development is a trend from wholistic to differential perception (Gibson 1969; Vurpillot 1976; Werner 1961; Wohlwill 1962). Several researchers (Shepp \& Swartz 1976; Smith \& Kemler 1977) have derived a corollary to this principle from Lockhead's $(1966,1972)$ and Garner's $(1970,1974)$ distinction between integral and separable perception. According to this derivation, development proceeds from wholistic perception organized by overall similarity to differentiated perception organized by dimensions. The new claim, contrary to earlier conceptualizations, is that wholistic perception is not diffuse and unstructured. Rather, wholistic perception is as organized as differentiated perception, but it is organized differently. A number of findings support the corollary (Kemler \& Smith 1978; Shepp 1978; Shepp \& Swartz 1976; Smith \& Kemler 1977, 1978). One result, obtained by Smith and Kemler (1977), will be discussed to clarify the proposed trend and to introduce the issue of present concern, namely, the importance of changes in perceptual organization for the development of classification skilis.
Smith and Kemler (1977) gave children sets of objects that varied in size and brightness and asked them "to put together the ones that go together." The important finding was that young children constructed groups highly organized by a wholistic relation. Objects were classified such that the objects in one group were maximally similar on both dimensions and the objects in separate groups were maximally dissimilar on both dimensions. For example, a 1-inch white square and a $1 / 2$-inch light grey square would be classified together. These two objects are not identical on either dimension, but they are highly similar on both. A white square and a black square of identical size would not be grouped together. Despite the size identity, these two objects are highly dissimilar overall. Older children also constructed organized groups but by the more traditional relation of one-dimensional identity. Objects were grouped together that were exactly alike on one dimension, and objects were grouped apart that differed on that same dimension. For example, the older child would group a 1-inch white square and a 1-inch black square together

The assistance of Catherine Kilroy and Timothy Henderson in the collection of the data and the cooperation of the staff and children at the Stinesville Elementary School is gratefully acknowledged. The comments and suggestions of Peter Jusczyk, Clark Presson, James Craig, and Richard Aslin are also appreciated. This research was supported by grant no. BNS 78-13019 from the National Science Foundation and by a Biomedical support grant through Indiana University. Reprint requests should be sent to Linda B. Smith, Department of Psychology, Indiana University, Bloomington, Indiana $\mathbf{4 7 4 0 5}$.

[Child Development, 1979, 50, 705-715. (C) 1979 by the Society for Research in Child Development, Inc. 0009-3920/79/5003-0011\$01.02] 
but apart from a $\frac{1}{12}$-inch grey square. Thus, both older and younger children's classifications were highly organized. It was the kind, and not the degree, of structure that depended on developmental level.

This interpretation of the young child's classifications as being organized contrasts with other interpretations. A "proper" classification is traditionally defined as one in which objects are partitioned into mutually exclusive groups according to some criterial property, for example, by identity on a dimension (e.g., Inhelder \& Piaget 1964). Under this definition, young children do classify poorly (e.g., Denney 1972; Inhelder \& Piaget 1964; Kofsy 1966; Vygotsky 1962). However, such results are also consistent with the present view. Dimensional classifications by young children are unexpected because perceiving dimensional relations takes processing time and effort at early developmental levels (e.g., Shepp \& Swartz 1976; Smith \& Kemler 1978). It is the characterization of nondimensional classifications as "illogical" and unstructured (e.g., Inhelder \& Piaget 1964) that is contested. Wholistic perception affords a systematic, although nondimensional, means for categorizing objects. Objects are grouped together because they are, overall, very much alike, and objects are grouped apart because they are, overall, dissimilar. Such a classification seems no less organized than one in which objects are grouped together because they are exactly alike in one way.

Furthermore, several researchers have suggested that overall similarity is the structure underlying many natural categories (see Rips, Shoben, \& Smith 1973; Rosch 1973; Rosch, Mervis, Gray, \& Johnson 1976). For example, chairs are said to be not all exactly alike in any one way but rather highly similar across many dimensions. In this context, the child's similarity classifications seem quite proper and sensible. Such a classification scheme may, in fact, be more useful than a dimensional one at early developmental levels.

However, the ability to classify according to dimensional identity is an important achievement, one that may be critical for the development of sophisticated problem solving. The theorists (e.g., Inhelder \& Piaget 1964; Vygotsky 1962) who deemed criterial property classifications proper did so for a reason. Identity classifications are basic to formal reasoning about sets and inclusion relations. Dimensional identity relations also have an advantage over wholistic similarity on an "everyday" level. It is certainly easier to verbalize and test the hypothesis that "all and only cows have an udder" than to state precisely and test the hypothesis that "most cows are mostly alike." In fact, adults are highly biased to search for dimensional identities in hypothesis-testing situations (Kemler \& Smith 1979). Thus, the dimensional classifications of older children and adults may be motivated by an intention to group according to an easily tested rule.

The purpose of the present study is to examine further the development of classificatory organizations. The child's task is to extend an already existing classification. The principled extension of a classification has been considered beyond the young child's ability (Inhelder \& Piaget 1964). However, by the present analysis, the young child should well be able to extend a similarity classification. This categorygeneralization task also provides an excellent measure of the systematic use of differentiated and wholistic relations. If a child defines a category by a value on one dimension, then he should include in that category all and only the objects which possess the criterial value. In contrast, if a category is defined by wholistic similarity, then all those objects and only those objects that are similar overall to existing members should be included.

In the first two experiments, children's spontaneous extensions of a classification are examined. In the third experiment, children's extensions are examined under hypothesis-testing instructions. The question of this final experiment is whether young children search for dimensional identities when their task is the discovery of a testable rule.

\section{General Method}

The basic task in all the experiments is as follows: The child is shown two groups of objects and told that the items in each group belong together. The child is then asked where he thinks new test items go: in the first group, in the second group, or not at all. The structure of the experimenter-formed groups and the test items are critical and are shown schematically in figure 1.

The stimulus items are composed of perceptually ordered values along two dimensions. Thus, each unique item is represented as a point in a two-dimensional space. The coordinates of the point indicate the component values on the two varying dimensions ( $X$ and $Y$ ). The solid circles indicate the items that form the two 


\section{Stimulus Sets for Experiments I and III}
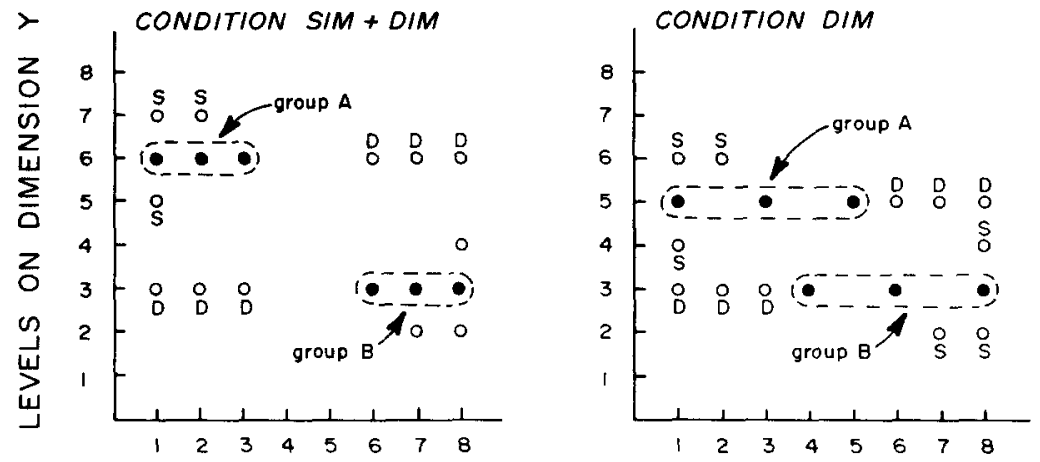

LEVELS ON DIMENSION $X$

FIG. 1.-Schematic representations of stimulus sets employed in experiments I and III. Coordinates of circles indicate values combined on the two varying dimensions to form the individual stimulus items in condition $S I M+D I M$ and in condition $D I M$. The items that form the two exemplar groups $(\mathrm{A}+\mathrm{B})$ in each condition are designated by solid circles; the $S$ and $D$ test items are designated by open circles.

exemplar groups (A and B), and the open circles indicate the new test items-the items whose membership in A and B are to be evaluated by the child. In condition $S I M+D I M$ (left-hand side of the figure), the exemplar classification is similarity maximizing and dimensional. It is a good similarity classification because the members of one group are "close" to each other and are "far" from the members of the second group. It is a good dimensional classification because all members of a group possess the identical value of dimension $Y$. However, the exemplar classification in condition DIM (right-hand side of the figure) is only well organized by dimensional relations. This classification is not similarity maximizing because the "distances" between members of the same group are often as large as the "distances" between members of separate groups. However, the classification is according to identity on one dimension $(Y)$. The test items are labeled according to the relation by which they might be included in the exemplar group: $S$ items are similar overall to the exemplar members, and $D$ items share the "criterial" value with the exemplar items.

Condition $S I M+D I M$ provides a measure of the preference of one structure over another. If the similarity structure is perceived, $S$ items should be included in the group to which they are nearest as they maintain the similarity-maximizing organization of the set. However, $D$ items should not be included in either exemplar group because these items are dissimilar, overall, to the members of both groups. In contrast, if the dimensional structure is perceived, $D$ items should be included according to their value on the criterial dimension and $S$ items which do not possess the criterial value should not be included in either group. Condition DIM provides a measure of the child's ability to apprehend the dimensional structure of a classification when there is no (good) similarity solution. If the dimensional structure is apprehended, $D$ items should be included by their value on the criterial dimension and $S$ items should be excluded from both groups.

\section{Experiment I}

\section{Method}

Subjects.-The subjects were 16 kindergartners, 16 second graders, and 16 fifth graders attending a rural Indiana elementary school. Mean ages at the three grade levels are 5-4 (range: 5-0 to 6-1), 7-6 (range: 7-2 to 7-11), and 10-6 (range: 10-0 to 11-2).

Stimuli and design.-The stimuli consisted of isosceles triangles of equal area (1 square inch) that varied in height (and thus also in base width) and color. The eight height values were: (1) $\frac{1 / 2}{2}$ inch, (2) $\%$ inch, (3) 3 inch, (4) 7\% inch, (5) 1 inch, (6) $1 \%$ inch, (7) $1 \%$ inch, and (8) 2 inches. Phenomenally, the triangles vary in shape from "short-fat" ones to "tallskinny" ones. The color of the triangles varied 
in eight steps from yellow to red; coloraid notation: (1) Y, (2) YOY, (3) YO, (4) OYO, (5) $\mathrm{O}$, (6) ORO, (7) ROR, (8) R. All differences were highly discriminable to adult observers and to kindergartners by a "same-different" oddity task.

Values of color and shape were combined to form the $S I M+D I M$ and $D I M$ stimulus sets, as shown in figure 1. Two additional items -not shown in the figure-were included in the test series. These items are located at coordinates 8,8 and 1,1 and are "don't go" itemsitems that should not be included in exemplar groups A or B by either a similarity or a dimensional rule. Four unique stimulus sets were constructed: (1) $S I M+D I M$, dimension $X$ equals color, (2) $S I M+D I M$, dimension $X$ equals shape; (3) DIM, dimension $X$ equals color; and (4) DIM, dimension $X$ equals shape.

Each stimulus set consisted of 30 items. Six of the items form the two exemplar groups (solid circles in fig. 1). The remaining 24 items -assembled in two random orders-form the test series and are of four types: six $D$ itemsthe items that should be included by a criterial dimension rule; six $S$ items-the items that should be included by a similarity rule; six $X$ items-three replications of the two "don't go" items; and six $E$ items-replications of each of the six exemplar items that form groups $A$ and B.

Half the children at each grade level were assigned to condition $S I M+D I M$ and half to condition DIM. Within each of these groups of children, half were assigned to each level of dimension $X$ ( $X=$ color, $X=$ shape). Order of test items was completely counterbalanced within each factor.

Procedure.-The experimenter faced the child across a table and sorted out the six exemplars into two groups. The child was told that the ones on the right "belonged together" and that the ones on the left "belonged together." The experimenter then told the subject that there were "a lot of other pictures" that had become mixed up by a mishap. The child was then asked where he thought these (test) items ought to be put. It was emphasized that some test items belonged in group $A$, that some belonged in group B, but that others did not belong at all and should be thrown out. One
$X$ item was shown to the child to illustrate this third "don't go" possibility and by experimenter and subject "agreement" was put in a basket on the floor.

Each test item was judged individually. Items judged as belonging in a group were laid face down near that group. Items judged as not belonging in either group were put in the basket. The exemplar groups remained separated and in view at all times. The child was periodically encouraged, but no feedback was given. An experimental session lasted approximately 25 min.

\section{Results and Discussion}

The $E$ and $X$ items in the test series are controls to ensure that the children were not including items haphazardly-without considering the members of the exemplar groups. At each grade level, the children included virtually all $E$ items in the appropriate groups and excluded virtually all $X$ items (mean proportion correct: .99 and .95 , respectively). The second preliminary measure is an examination of the frequency with which items are included in a manner inconsistent with either the similarity or the dimensional rule. For example, if a specific $D$ item shares a value with the exemplars of group A, it should be classified with group A or judged as not belonging in either group; it should not be included in group B by either rule. Judgments inconsistent with either a similarity or dimensional rule occurred rarely (mean proportions: .02 for kindergartners and 0 for second and fifth graders). Thus, in the subsequent analyses, individual inclusions of $S$ and $D$ items are (arbitrarily) scored right or wrong by the dimensional rule; that is, inclusion of a $D$ item is "correct," and noninclusion of an S item is "correct." An "incorrect" judgment, of course, is correct by a similarity-maximizing rule.

Each subject was scored separately on $D$ and $S$ items. These scores were submitted to a 3 (grade) $\times 2$ (condition) $\times 2$ (dimension $X) \times 2$ (item type) analysis of variance for a mixed design. 1 The analysis yielded main effects of grade, $F(2,36)=11.30, p<.001$, condition, $F(1,36)=5.15, p<.001$, and item type, $F(1,72)=34.00, p<.001$. Mean proportion correct by the dimensional rule on $S$ and $D$ items at each age level and in each condition is shown in table 1 .

1 All analyses of the data obtained in the three experiments were computed on frequencies and not proportions, since a number of subjects contributed proportions close to or equal to one and zero. The data are presented in terms of proportions in the tables, however, as such scores more easily illustrate the consistency of use of a dimensional or similarity principle. 
TABLE 1

Mean Proportion Correct by the DtmenSTONAL RULE IN EXPERIMENT I

\begin{tabular}{|c|c|c|c|c|}
\hline \multirow[b]{3}{*}{ GRADE } & \multicolumn{4}{|c|}{ CONDITION } \\
\hline & \multicolumn{2}{|c|}{$S I M+D I M$} & \multicolumn{2}{|c|}{$D I M$} \\
\hline & $\begin{array}{l}D \text { (In- } \\
\text { cluded) }\end{array}$ & $\begin{array}{c}S \\
\text { (Not In- } \\
\text { cluded) }\end{array}$ & $\begin{array}{l}D \text { (In- } \\
\text { cluded) }\end{array}$ & $\begin{array}{c}S \\
\text { (Not In- } \\
\text { cluded) }\end{array}$ \\
\hline $\mathrm{K}$. & .33 & .37 & $\begin{array}{c}.73 \\
(38)\end{array}$ & .40 \\
\hline 2. & .56 & .33 & .90 & .58 \\
\hline & $(.38)$ & $(.35)$ & $(.12)$ & $(.33)$ \\
\hline 5. & $\begin{array}{l}.89 \\
(.33)\end{array}$ & $\begin{array}{l}.69 \\
(.35)\end{array}$ & $\begin{array}{l}.99 \\
(.06)\end{array}$ & $\begin{array}{l}.89 \\
(.19)\end{array}$ \\
\hline
\end{tabular}

NoTE.-SDs in parentheses.

Consider first performances in condition $S I M+D I M$. Post hoc comparisons ( $\alpha=.05$, Tukey's method corrected as per Cichetti [1972]) reveal that $D$ items are appropriately included more often at each increasing age level and that $S$ items are judged as not belonging reliably more often by fifth graders than by the younger children. This trend is consistent with the apprehension of qualitatively different category structures at different age levels. Kindergartner's classifications conform to the similarity organization reliably more often than expected by chance, two-tailed, $t(7)=$ $2.38, p<.05$. Fifth graders, on the other hand, classified dimensionally, two-tailed $t(7)=4.62$, $p<.01$. The second-grade pattern of performance, however, does not differ from that expected by chance alone, $t(7)<1.00$. An examination of individual performances by second graders suggests that these subjects are in transition between the use of the similarity and the dimensional structure. Four second grader's classifications were consistent with the similarity principle at least $67 \%$ of the time. Three second graders classified by the dimensional principle at least $67 \%$ of the time. (The remaining second grader included items in a manner consistent with each structure exactly $50 \%$ of the time.) Thus, both older and younger children systematically extend a classification. However, the system of choice for young children is wholistic similarity, whereas for older children it is identity on a criterial dimension.

The interpretation of the pattern of performance in condition $D I M$ is less clear cut. If the classification of $S$ and $D$ items is jointly considered, the use of the dimensional structure appears stronger in this condition relative to that in condition $S I M+D I M-$ particularly at the younger age levels. However, as can be easily seen in the right-hand side of table 1 , these increases in dimensional classifications are due primarily to the inclusion of more $D$ items and not to an increase in the noninclusion of $S$ items.

One interpretation of this asymmetry between scores on $D$ and $S$ items (which is also, but more weakly, apparent in condition SIM + $D I M)$ is that deciding an item does not belong is more difficult than deciding it does. In other words, when a subject is in doubt about the classification of a particular item-a state likely to be prevalent in younger children in condition $D I M$-he decides to include it rather than "throw it out." The notion is that when there is not a good similarity solution, young children access the dimensional organization, but doing so is somewhat difficult for them.

A second interpretation is also possible. The younger children may not systematically classify unless there is a possible similarity solution. In condition DIM, some kindergartners tended to state that a test item "belonged with" a particular exemplar item. The child would say, for example, "it goes with this one," picking up the one exemplar item to which the test item was most similar. By examining figure 1, it is evident that such an object-to-object matching scheme would result in the inclusion of many $D$ and $S$ items. In condition $D I M$, then, young children may not conceptualize the two exemplar groups as categories governed by a particular organization. Rather, they may apprehend a set of six diverse objects and in the test series match one specific item to another. This interpretation is highly reminiscent of Inhelder and Piaget's (1964) "de proche en proche" characterization of young children's classifications.

This experiment indicates that young children can generalize a classification in a systematic manner by wholistic similarity. Older children, however, generalize by one-dimensional identity. The ability of young children to extend a category by value on one dimension appears weak and is examined more closely in experiment II.

\section{Experiment II}

In this category-generalization task, the explicit noninclusion of an item was not required. The child was only asked which of several new items "belonged" in the exemplar 
group. These new test items were structured to diagnose the use of a similarity organization, a dimensional organization, or an object-toobject matching strategy.

The structures of the stimulus sets are shown in figure 2. In condition $S I M+D I M$ the exemplar items $\left(E_{1}\right.$ and $\left.E_{2}\right)$ share a value on one dimension $(X)$ and are highly similar on the other $(Y)$. The three types of test items are (1) $S$ items-highly similar to the exemplars overall; (2) $D$ items-identical on the criterial dimension but dissimilar overall; and (3) $M$ items-highly dissimilar overall. The exemplar set in condition DIM differs from that in condition $S I M+D I M$ by only one stimulus; the structure of the set, however, is quite different. The exemplar items share a value on one dimension $(X)$ and are relatively dissimilar on the other $(Y)$. The three test items are (1) $S$ items-similar to one member of the exemplar pair and somewhat similar to the other member; (2) $D$ items--identical on the criterial dimension and dissimilar to the exemplars overall; and (3) $M$ items-highly similar to one member of the exemplar pair and highly dissimilar to the other.

The expectation is that with SIM + DIM sets the kindergartners will choose to include the item (S) most similar overall to both exemplars. With DIM sets the possibilities of interest are that the kindergartners will (1) include the item $(D)$ consistent with a dimensional organization or (2) include the items ( $S$ and $M$ either equally often or perhaps $M$ slightly more often) that are similar to one member of the pair (an object-to-object matching strategy). No specific predictions are made about second graders' performance because second graders as a group appear to be more sensitive to dimensional relations than kindergartners but still somewhat influenced by wholistic similarity.

\section{Method}

Subjects.-Eight kindergartners and eight second graders who were classmates of the children participating in experiment I served as subjects. Mean ages were 5-6 (range: 5-2 to 6-0) and 7-6 (range: 7-1 to 8-1).

Stimuli and design.-All stimulus sets were chosen from the same $8 \times 8$ two-dimensional space described in experiment I. The eight unique $S I M+D I M$ sets were structured as follows (see fig. 2): The two exemplar $(E)$ items shared a value on dimension $X$ and differed by one step on dimension $Y$; test item $S$ differed by one step on both dimensions from one exemplar, $E_{1}$, and differed only by one step on dimension $Y$ from $E_{2}$; test item $D$ shared a value with $E_{1}$ and $E_{2}$ on $X$ and differed by four steps (from $E_{1}$ ) on $Y$; test item $M$ differed from $E_{2}$ by one step on $X$ and two steps on $Y$. Each of the eight unique DIM sets was identical with a $S I M+D I M$ set except for value $Y$ on $E_{2}$, which differed from $E_{1}$ by three steps in such a way that $E_{2}$ was made more similar to item $M$ (a one-step difference on $X$ ) and less similar to item $S$ (a two-step difference on $Y$ ).

In four of the $S I M+D I M$ sets, and there-

\section{Stimulus Sets for Experiment II}
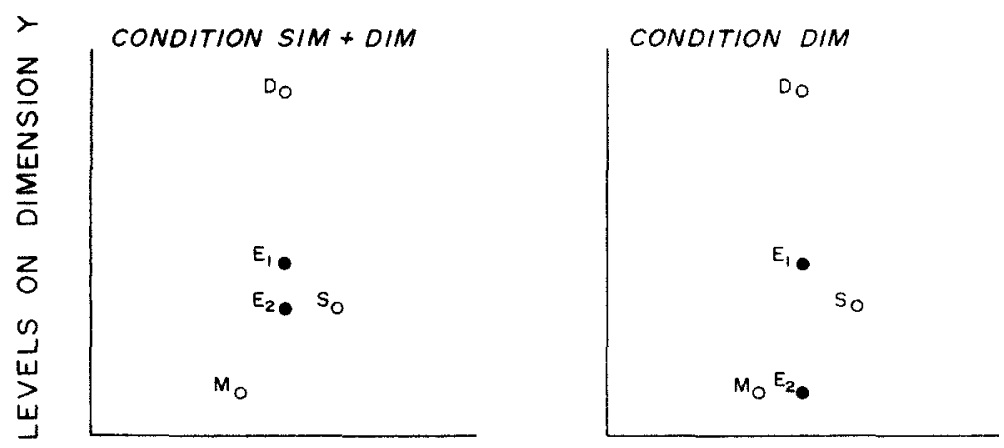

\section{LEVELS ON DIMENSION $X$}

Fic. 2.-Schematic representations of the two types of stimulus sets-SIM $+D I M$ and $D I M$-employed in experiment II. Individual items are represented as in figure 1. The exemplar items are designated by solid circles and the three test items $-D$, $S$, and $M$-by open circles. 
fore in four of the DIM sets, dimension $X$ was color; on the remaining sets dimension $X$ was shape. Each child judged each of the 16 sets (8 SIM + DIM sets and $8 D I M$ sets) in one of two random orders.

Procedure.-The child was shown the two exemplar items and was told that they belonged together. The three test stimuli were then placed above the exemplar group. The child was asked which one of the three he thought most belonged with the two exemplars. This procedure was repeated for the 16 trials. No specific feedback was given. A session lasted approximately $20 \mathrm{~min}$.

\section{Results and Discussion}

Kindergartners' performance.-The first expectation is that when there is a similarity solution to a category-inclusion problem, young children will use that relation. This expectation was confirmed. As can be seen in the top left of table 2, the test item (S) that is highly similar to both members of the exemplar set is chosen more often than the other test items, $t(7)=$ $6.00, p<.001$. Again, the relation of choice for the young child appears to be wholistic similarity.

What do these same children do when the exemplar category does not offer a good similarity organization? As can be seen in the top right of table 2, kindergartners' choices on DIM trials appear to conform to no consistent rule. The choice of test items is, in fact, not distributed differently from that expected by chance, $t(7)<1.00$. This characterization fits the pattern of individual performances as well. Only two of the eight kindergartners consistently included items of a certain type on DIM trials: one individual according to the objectto-object strategy, the other according to value on one dimension.
Whereas kindergartners spontaneously and consistently generalize from a category to new instances by wholistic similarity, their inclusions appear unsystematic when the category is organized dimensionally. The finding that kindergartners do not use an object-to-object matching strategy on DIM trials is of some importance. This strategy has been suggested to be one that is characteristic of young children (Bruner, Olver, \& Greenfield 1966; Inhelder \& Piaget 1964). Some kindergartners in some category-generalization situations (e.g., as in condition DIM of experiment I) may resort to a matching strategy when other preferred solutions are not available. However, the combined results of experiments I and II suggest that an object-to-object matching strategy is not general. Rather, haphazard classifications appear to be the rule when the stimulus set is not well organized by wholistic similarity.

Second graders' performance.-As a group, second graders, again, appeared to be more sensitive to the dimensional structure of a category than kindergartners. Although inclusions of $S$ and $D$ items on the $S I M+D I M$ trials do not differ reliably from each other, $t(7)<1.00$, systematic inclusions do characterize individual performances: four second graders chose the $S$ item at least $60 \%$ of the time and three chose the $D$ item $88 \%$ of the time. Moreover, on condition DIM trials (when there is not a good similarity solution), all eight subjects chose to include item $D$ at least $60 \%$ of the time. As evident by examining the bottom right of table 2 , inclusion of item $D$ on the DIM trials predominated, occurring reliably more often than expected by chance alone, $t(7)=$ $4.81, p<.001$.

Second graders are certainly further along in the progression from similarity to dimensionally based perception than kindergartners. While the youngest subjects may be able to

TABLE 2

Mean Proportion of Inclusions in Experiment II

\begin{tabular}{|c|c|c|c|c|c|c|}
\hline \multirow[b]{3}{*}{ Grade } & \multicolumn{6}{|c|}{ Stimulus SeT } \\
\hline & \multicolumn{3}{|c|}{$S I M+D I M$} & \multicolumn{3}{|c|}{$D I M$} \\
\hline & $S$ & $D$ & $M$ & $S$ & $D$ & $M$ \\
\hline $\begin{array}{l}K \\
2 .\end{array}$ & $\begin{array}{l}.69 \\
(.23) \\
.48 \\
(.33)\end{array}$ & $\begin{array}{l}.28 \\
(.17) \\
.52 \\
(.33)\end{array}$ & $\begin{array}{c}.03 \\
(.06) \\
.00 \\
\ldots\end{array}$ & $\begin{array}{l}.24 \\
(.17) \\
.20 \\
(.18)\end{array}$ & $\begin{array}{l}.45 \\
(.23) \\
.68 \\
(.23)\end{array}$ & $\begin{array}{l}.31 \\
(.23) \\
.12 \\
(.15)\end{array}$ \\
\hline
\end{tabular}

Note.-SDs in parentheses. 
perceive and use component dimensions in some task contexts (Kemler \& Smith 1979; Smith \& Kemler 1978), their level of sophistication is not such that they spontaneously apprehend a category as organized by a one-dimensional identity. In contrast, all second graders easily extend a category by its dimensional structure, at least when there is not a competing similarity organization.

The major conclusion from the first two experiments is that both younger and older children can systematically extend a category. It is only the organizations behind the extensions that differ.

\section{Experiment III}

In this final category-generalization task, the child is provided with a possible motivation for accessing the dimensional structure of a classification. The young child is asked to discover a classification rule. If dimensional rules are more easily tested than similarity rules, then the young child as well as the older one might attend to and learn about dimensions in this task. Again, classifications well organized by both wholistic similarity and dimensional identity $(S I M+D I M)$ are contrasted with ones only well organized by a dimensional relation (DIM).

\section{Method}

Subjects.-The subjects were 16 kindergartners and 16 second graders attending the same elementary school as the children who participated in experiments I and II. Mean ages were 5-5 (range: 5-1 to 5-10) and 7-8 (range: 7-2 to 8-0).

Stimuli and design.-The stimulus sets (SIM + DIM and DIM) and design were identical with that in experiment I (see fig. 1).

Procedure.-The child was shown the six exemplar items sorted into the two groups and was told that the ones on the right belonged together for a special reason and that the ones on the left belonged together and apart from the others for a special reason. The child was then told to "find the special reason." The six exemplars were picked up and shuffled, and the child was asked to put them back into the two groups by the "rule." All sorting errors were corrected, but the child was not informed about the nature of the rule. The sorting of the exemplars continued until the child sorted correctly on five consecutive trials or for a maximum of 12 trials (failure criterion). Children who passed this phase of pretraining were then shown each test item and asked to indicate the appropriate classification as in experiment I (group A, group B, or "don't go"). After each judgment, the test stimulus was removed. No feedback was given. The exemplar items were in view throughout the session, which lasted approximately $30 \mathrm{~min}$.

\section{Results and Discussion}

All children but one subject (a kindergartner in condition DIM who was replaced) passed pretraining. Further, all children appropriately included the exemplar $(E)$ items and appropriately did not include the "don't go" items $(X)$ (mean proportions: .98 and .95 , respectively). Again, as in experiment I, inclusions not conforming to either a dimensional or similarity rule occurred extremely rarely (less than $2 \%$ of the inclusions). Therefore, classifications of $S$ and $D$ items are scored according to the dimensional rule in experiment 1 . Of course, "incorrect" classifications by this "dimensional" scoring scheme are correct by a similarity rule in condition $S I M+D I M$.

\section{Kindergartners' performance.-The ques-} tion in this experiment is what rule, if any, did the children discover. In condition SIM + DIM, the children could learn either a wholistic similarity rule or a dimensional rule. As can be seen in table 3 , kindergartners do not appear to have discovered (or learned) either nule. The mean number of kindergarten inclusions and noninclusions correct by a dimensional rule (and therefore by a similarity rule) does not differ reliably from that expected by chance, $t(7)=1.17$. This result stands in marked contrast to those of the first two experiments. In a free generalization task, kindergartners prefer and systematically use wholistic similarity. How-

TABLE 3

Mean Proportion Correct by the Dimensional RULE IN EXPERIMENT III

\begin{tabular}{|c|c|c|c|c|}
\hline \multirow[b]{3}{*}{ Grade } & \multicolumn{4}{|c|}{ Strmulus SET } \\
\hline & \multicolumn{2}{|c|}{$S I M+D I M$} & \multicolumn{2}{|c|}{$D I M$} \\
\hline & $\begin{array}{l}D(\text { In- } \\
\text { cluded) }\end{array}$ & $\begin{array}{l}\text { S } \\
\text { (Not In- } \\
\text { cluded) }\end{array}$ & $\begin{array}{l}D(\text { In- } \\
\text { cluded })\end{array}$ & $\begin{array}{c}S \\
\text { (Not In- } \\
\text { cluded) }\end{array}$ \\
\hline $\begin{array}{l}\mathbf{K} \ldots \ldots \\
2 \ldots \ldots\end{array}$ & $\begin{array}{l}.58 \\
(.40) \\
.73 \\
(.28)\end{array}$ & $\begin{array}{c}.52 \\
(.33) \\
.60 \\
(.33)\end{array}$ & $\begin{array}{c}.65 \\
(.36) \\
.90 \\
(.19)\end{array}$ & $\begin{array}{c}.69 \\
(.33) \\
.67 \\
(.36)\end{array}$ \\
\hline
\end{tabular}

Note, - SDs in parentheses. 
ever, when the children are required to learn a classification well organized by wholistic similarity, they abandon the wholistic relation. Only three of eight kindergartners' judgments were systematic by either rule, and for all three the rule was identity on one dimension. The pattern of kindergarten performance in condition $D I M$ also differs from the earlier results. The only general rule that could be discovered in this condition was one about dimensional identities, and all but one kindergartner discovered that rule. Thus, the mean number correct by a dimensional rule is reliably greater than chance, $t(7)=2.86, p<.05$.

Clearly, kindergartners' attention to the component dimensions of objects was increased by the pretraining task. Kindergartners can generalize a category according to a criterial dimension if a good similarity solution is not possible and if they are required to discover a general classification rule. Further, when strong similarity relations are available, kindergartners do not learn about that relation (even though they seem unable to completely ignore wholistic similarity).

Second graders' performance.-Again, second graders showed a greater ability to use a criterial dimension than kindergartners. In this rule-discovery task, new items were included by second graders only if they possessed the proper dimensional value. This rule was discovered both when there was a strong competing similarity organization and when there was not a competing solution (contrasted with scores expected by chance: $S I M+D I M, t[7]$ $=3.33, p<.05 ; D I M, t[7]=5.45, p<.001)$. Note, again, the contrast with the results of experiment I. In the free generalization task, a substantial number of second graders generalized by wholistic similarity when the solution was available. When required to discover a classification rule, however, no child abstracted a rule about wholistic similarity. At both levels of development, then, attention to dimensional relations is increased when the task requires the discovery of a classification rule.

\section{General Discussion}

Both younger and older children systematically extend a classification by the relation that dominates their perception. Wholistic similarity is perceptually primary at early levels of development, and the young child uses this relation to categorize objects. Dimensional relations are perceptually primary at later levels of development, and the older child uses these re- lations to classify. These findings offer new insight into the development of classification. Traditional studies focused on the child's use of dimensional relations and ignored other possible classification schemes. In such a context, the young child seemed not to understand the nature of classification (Inhelder \& Piaget 1964). However, the child's use of wholistic similarity shows that he can classify. The young child's categories, as well as those of the older child, are defined by an organization that determines category membership. In this sense, classification does not develop. It is the ability to classify by a certain relation, namely, identity on a dimension, that depends on developmental level.

However, the relation of dimensional identity appears psychologically special even at early developmental levels. In experiment III, we found that when young children learn a classification rule, they learn a rule about dimensional relations. This result is consistent with Gibson's (1969) position. Perceptual learning consists of the differentiation and isolation of invariant relations (i.e., dimensional identities). Still, the question of why young children learn about dimensional relations and not wholistic similarity is perplexing. On the one hand, wholistic similarity appears to be the perceptually dominant relation for young children, but on the other, young children do not abstract this relation in a learning task. Further, wholistic similarity is claimed to be a reasonable organization, but it is not one that is used by mature classifiers. This paradox may be explained by considering the possible psychological functions of similarity and dimensional relations.

The young child's sensitivity to wholistic similarity may serve to facilitate the formation of basic concepts. If categories such as "chair" are structured by overall similarity (see Rips et al. 1973; Rosch 1973), then the young child must perceive this relation if he is to organize the objects he encounters every day. One problem with criterial property accounts of natural concepts is that young children do have difficulty discovering identity relations (cf. Nelson 1974). Family-resemblance (similarity) accounts of natural concepts have no such problem. Wholistic similarity is a relation to which the child is perceptually tuned. However, wholistic similarity may be a perceptual relation and not easily available to conscious awareness. This wholistic relation may serve to organize only intuitive, unanalyzed categories. 
In contrast, the value of identity classifications may derive from the structure of human hypothesis testing. Psychologists' historic emphasis on criterial properties (cf. Markman \& Siebert 1976; Nelson 1974; Rosch 1973) highlights the special status of dimensional identities in scientific reasoning. In this context, categories structured by dimensional relations have many advantages over those structured by similarity. For example, the relation of dimensional identity is an equivalence relation, both transitive and symmetrical. However, similarity relations are not necessarily transitive or symmetrical. The formal description of similarity categories is much more complex than that of identity categories (see Tversky 1977). It is conceptually simpler to make a dimensional structure explicit than a similarity structure, and the reasoning power available from identity relations is greater than that from similarity relations. Thus, identity relations may serve to organize intentional classifications. When one needs an explicit rather than tacit classification rule, the identity relation is more useful.

This proposed account of the difference between wholistic similarity and identity relations shares much with Piaget's (1969; Inhelder \& Piaget 1964) distinction between perceptual and intellectual classification. Consistent with Piaget's analysis, the young child classifies intuitively and perceptually, whereas the older child classifies with plans and intention. However, by the present view, the young child's "intuitive" groupings are highly organized. Young children do not classify poorly but, rather, by a relation that is particularly useful to their major cognitive task. Older children may also classify by the relation that is most useful to their level of cognitive development. The direction of the trend seems reasonable. Children first attend to relations between whole functional objects; later they formulate and test rules about the component attributes of these objects.

\section{References}

Bruner, I. S.; Olver, R. R.; \& Greenfield, P. M. Studies in cognitive growth. New York: Wiley, 1966.

Cichetti, D. V. Extension of multiple-range tests to interaction tables in analysis of variance: a rapid approximate solution. Psychological Bulletin, 1972, 77, 405-408.

Denney, N. W. A developmental study of free classification in children. Child Development, 1972, $43,221-232$.
Garner, W. R. The stimulus in information processing. American Psychologist, 1970, 25, 350358.

Garner, W. R. The processing of information and structure. Potomac, Md.: Erlbaum, 1974.

Gibson, E. J. Principles of perceptual learning and development. New York: Appleton-CenturyCrofts, 1969.

Inhelder, P., \& Piaget, J. The early growth of logic in the child. New York: Norton, 1964.

Kemler, D. G., \& Smith, L. B. Is there a developmental trend from integrality to separability in perception? Journal of Experimental Child Psychology, 1978, 26, 498-507.

Kemler, D. G., \& Smith, L. B. Accessing dimensional and similarity relations: adults and young children's discovery of abstract relational concepts. Journal of Experimental Psychology: General, 1979, 108, 133-150.

Kofsy, E. A scalogram study of classificatory development. Child Development, 1966, 37, 191204.

Lockhead, G. R. Effects of dimensional redundancy on visual discrimination. Journal of Experimental Psychology, 1966, 72, 95-104.

Lockhead, G. R. Processing dimensional stimuli: a note. Psychological Review, 1972, 79, 410419.

Markman, E. M., \& Seibert, J. Classes and collections: internal organization and resulting holistic properties. Cognitive Psychology, 1976, 8, 561-577.

Nelson, K. Concept, work, and sentence: interrelations in acquisition and development. Psychological Review, 1974, 81, 267-285.

Piaget, J. The mechanisms of perception. New York: Basic, 1969.

Rips, L. E.; Shoben, E.; \& Smith, E. Semantic distance and the verification of semantic relations. Journal of Verbal Learning and Verbal Behavior, 1973, 12, 1-20.

Rosch, E. H. On the internal structure of perceptual and semantic categories. In T. E. Moore (Ed.), Cognitive development and the acqutsition of language. New York: Academic Press, 1973.

Rosch, E.; Mervis, C. B.; Gray, W. D.; Johnson, D. M.; \& Boyes-Braem, P. Basic objects in natural categories. Cognitive Psychology, 1976, 8, 382-439.

Shepp, B. From perceived similarity to dimensional structure: a new hypothesis about perceptual development. In E. Rosch and E. B. Lloyd (Eds.), On the nature and principle of formation of categories. Hillsdale, N.J.: Erlbaum, 1978.

Shepp, B. E., \& Swartz, K. B. Selective attention and the processing of integral and nonintegral 
dimensions: a developmental study. Journal of Experimental Child Psychology, 1976, 22, 7385.

Smith, L. B., \& Kemler, D. G. Developmental trends in free classification: evidence for a new conceptualization of perceptual development. Journal of Experimental Child Psychology, 1977, 24, 279-298.

Smith, L. B., \& Kemler, D. G. Levels of experienced dimensionality in children and adults. Cognitive Psychology, 1978, 10, 502-532.

Tversky, A. Features of similarity. Psychological Review, 1977, 84, 327-352.
Vurpillot, E. The visual world of the child. New York: International Universities Press, 1976.

Vygotsky, L. S. Thought and language. Cambridge, Mass.: M.I.T. Press, 1962.

Werner, H. Comparative psychology of mental development (Rev. ed.). New York: Scientific Editions, 1961.

Wohlwill, J. From perception to inference: a dimension of cognitive development. In W. Kessen \& C. Kuhlman (Eds.), Thought in the young child. Monographs of the Society for Research in Child Development, 1962, 27(2, Serial No. 83), 87-107. 
This document is a scanned copy of a printed document. No warranty is given about the accuracy of the copy. Users should refer to the original published version of the material. 